\title{
3D Imaging with Single Atom Sensitivity using Confocal STEM
}

K. van Benthem, N. de Jonge, A.Y. Borisevich, M.P. Oxley, S.J. Pennycook Oak Ridge National Laboratory, Oak Ridge, TN 37831-6031, USA

Aberration correction in scanning transmission electron microscopy (STEM) enables the formation of electron probes with diameters $\delta_{\mathrm{s}}$ of $1 \AA$ or smaller by significantly increasing the illumination angle $\alpha[1,2]$. As a consequence, the imaging of single atoms within materials or on their surfaces has become routine practice. Another benefit of aberration correction is that larger illumination angles also drastically decrease the optical depth of focus $T$. This is visualized for an uncorrected and an aberration corrected VG Microscopes' HB603U STEM in Figures 1 and 2. Figure 2 clearly shows that with a corrected electron probe thinner slices of the sample come into focus. Under purely geometrical considerations in the absence of lens aberrations and electron channeling, the depth of focus can be written as

$$
T=\frac{\delta_{s}}{\alpha}=\frac{\lambda}{\alpha^{2}} .
$$

A through-focal series acquisition effectively leads to depth-slicing of the sample, so that a slice-by-slice image of the three-dimensional structure can be obtained. After aligning the recorded images with respect to each other to compensate for any spatial drift during the acquisition, the recorded image stacks can be used for 3-D reconstructions for further investigations of the true three-dimensional structure of, e.g., interfaces and grain boundaries.

In favorable situations, vertical localization of single atoms in semiconductors (see Figure 3) and catalyst materials can be determined to a precision much higher than the vertical resolution, provided a sufficient signal to noise ratio is achieved [3-4].

Crystals in a zone axis orientation show strong channeling effects which complicate the depth sectioning. These effects are stronger for columns of higher atomic number and for smaller probe convergence angles. With future generations of correctors, zone-axis depth sectioning will become feasible is some materials.

\section{References:}

[1] P.E. Batson, N. Dellby, O.L. Krivanek, Nature 418, 617-620 (2002).

[2] P.D. Nellist et al., Science 305, 1741 (2004).

[3] K. van Benthem et al., Appl. Phys. Lett. 87, 034104 (2005), Ultramicroscopy (2006).

[4] A.Y. Borisevich et al., PANS in press (February 2006). 


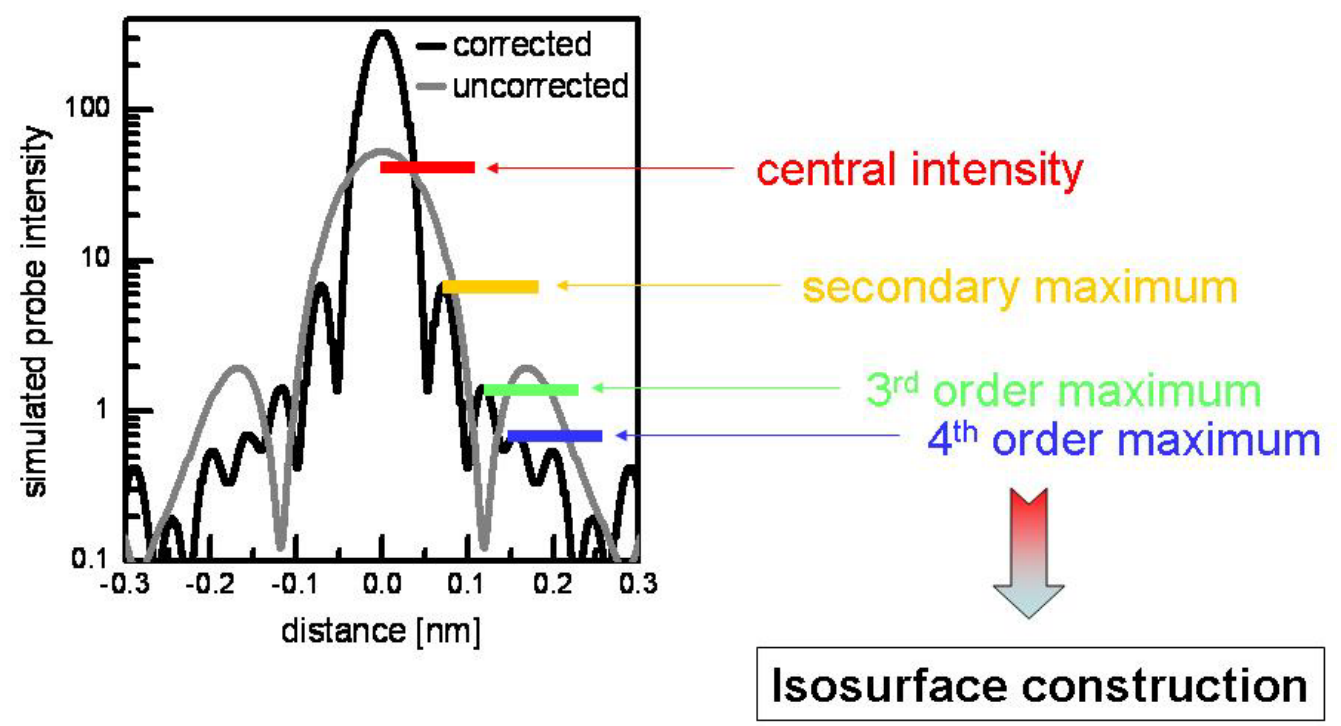

Figure 1: Simulated electron probe profiles for an uncorrected and an aberration corrected VG HB603 U dedicated STEM.

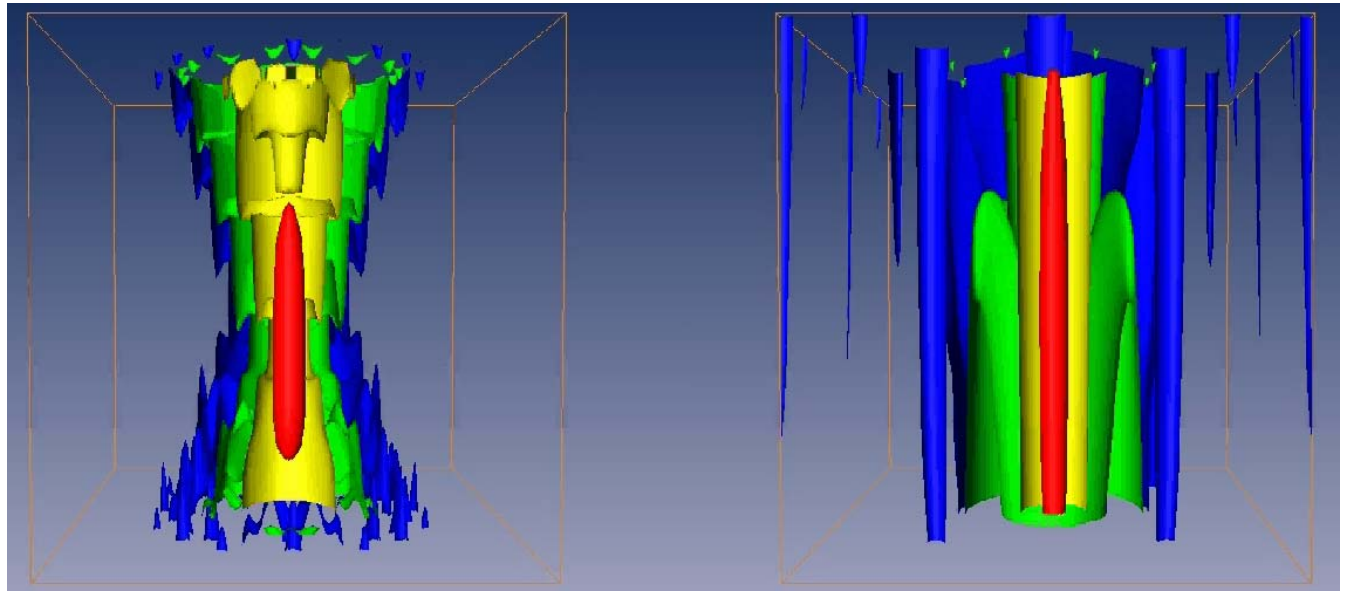

Figure 2: $\quad 3 \mathrm{D}$ reconstruction of the electron probe intensities for both the corrected (left) and uncorrected (right) cases as a function of defocus. Intensity levels are color coded according to Figure 1.

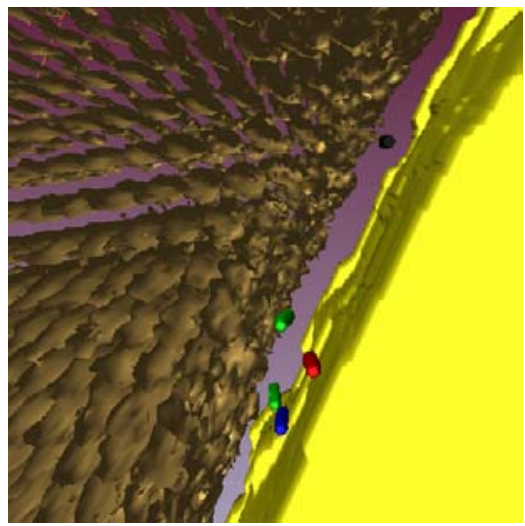

Figure 3: $3 \mathrm{D}$ reconstruction of a $\mathrm{Si} / \mathrm{SiO}_{2} / \mathrm{HfO}_{2}$ interface with single $\mathrm{Hf}$ atoms present inside the $\mathrm{SiO}_{2}$ interlayer. 